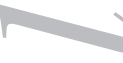

\title{
Factores genéticos en la enfermedad grasa del hígado no alcohólica
}

En la actualidad nadie duda del papel primordial que juegan los factores ambientales en la patogenia de la enfermedad grasa del hígado no alcohólico (EGHNA). La vida sedentaria, la alimentación abundante y rica en calorías, grasas sobresaturadas, carne, hidratos de carbono y fructosa y pobres en antioxidantes y en ácidos grasos $\omega$-3 (1-3) son factores esenciales en el desarrollo del llamado síndrome metabólico (obesidad central, hipertrigliceridemia, descenso del colesterol ligado al HDL, hipertensión arterial, hiperglucemia) (4,5) y, dentro de este, la EGHNA (6). A pesar de ello, es muy probable que otros factores individuales puedan también intervenir, determinando que la existencia y la gravedad de la EGHNA varíe mucho de unos individuos a otros y que sólo una minoría de personas con esteatosis hepática evolucione a esteatohepatitis no alcohólica (EHNA) y desarrolle fibrosis, cirrosis hepática e incluso carcinoma hepatocelular. En efecto, el estudio histológico del hígado de pacientes con obesidad mórbida demuestra que la existencia y gravedad de la EGHNA varía mucho de unos sujetos a otros. Por ejemplo, en el estudio de García Monzón y cols., realizado en 46 obesos sometidos a cirugía bariática, se encontró que el hígado carecía de lesiones en el $8,7 \%$, que había sólo un hígado graso en otro $22 \%$ y EHNA en el 69,5\%, la mayoría de estos con fibrosis (7). Resultados similares se encuentran en otros múltiples trabajos en los que se han analizado las lesiones hepáticas existentes en los obesos y en otras situaciones clínicas asociadas al síndrome metabólico (8-11). Por ello, muchos autores se han planteado el papel que pudieran jugar los factores genéticos en la patogenia de la EGHNA (12). A favor de ello aboga la existencia de varios casos de EGHNA en una misma familia (13-15) o el hecho de que en algunos países asiáticos (Japón, China, Filipinas, etc.) esta enfermedad sea especialmente frecuente o que, en los Estados Unidos, la EGHNA sea mucho más frecuente entre los hispanos, nativos americanos y asiáticos, todos ellos con una raíz étnica común, que entre los caucásicos y afroamericanos $(16,17)$.

No se conocen aún cuáles son los genes responsables de esta supuesta predisposición genética, sin embargo, la disponibilidad de nuevas técnicas de análisis genómico está proporcionando datos que nos están ayudando a conocer las bases hereditarias de la EGHNA. En el presente número de la Revista Española de Enfermedades Digestivas (18), Cayón y cols., empleando tecnología de macroarray, presentan el perfil de expresión genética en el hígado de un grupo de pacientes con EHNA y obesidad mórbida sometidos a cirugía bariática. Estos autores encuentran que los obesos tienen una sobreexpresión de genes relacionados con la respuesta inflamatoria [TNF $\alpha$, TNF $\alpha \mathrm{RI}$ (TNF $\alpha$ receptor I); M-CSF (macrophage colony-stimulating factor)], con la apoptosis [TRAIL-R2 (TNF-related apoptosis-inducing ligand receptor 2)] y con la protección de los complejos enzimáticos mitocondriales frente a su degradación (prohibitina). Los hallazgos de este grupo de investigadores son 
concordantes con el hecho de que los pacientes con EHNA presenten una actividad inflamatoria hepática que no existe en los pacientes con hígado graso simple y con los hallazgos de diversos autores que muestran que las tasas de citoquinas proinflamatorias se encuentran elevadas tanto en los pacientes con EHNA como en animales con EHNA experimental (19-24). Además, se han encontrado polimorfismos en el promotor del $T N F \alpha$ (25) y en el del receptor de la endotoxina CD14, que están asociados con un aumento de la producción celular de TNFo (26). El aumento de la expresión de TRAIL-R2 sugiere que la muerte celular por apoptosis es también un mecanismo de daño hepático en esta enfermedad. Las vías por las que se puede producir este tipo de muerte en la EHNA son diversas, pero entre ellas se ha implicado al TNF $\alpha$, los ácidos grasos libres y el estrés oxidativo (27-29). Estos hallazgos de Cayón y cols. indican que, además de los anteriores, también TRAIL pudiera participar en ese proceso. Aunque no está completamente demostrado que este factor provoque la muerte de los hepatocitos sanos, sí es muy probable que esta vía intervenga en la muerte de células previamente anormales (30). El aumento de la expresión genética de la prohibitina, complejo proteico con función protectora mitocondrial, probablemente tiene carácter reactivo a su rápida degradación. Nosotros y otros autores hemos comprobado que en el hígado de los pacientes con EHNA y en los ratones obesos la cantidad de esta proteína se encuentra muy disminuida $(31,32)$, lo cual es atribuible a su degradación mitocondrial como consecuencia de su nitración por el peroxinitrito (33). La exposición de las membranas mitocondriales a este radical provoca la nitración de esta proteína, su degradación y desaparición, mientras que el tratamiento de ratones obesos con ácido úrico, un captador de peroxinitrito, evita la nitración de la prohibitina y el descenso de su cuantía (31). Se puede entender que, ante la agresión que sufre la prohibitina, el organismo responda aumentando su expresión genética y su síntesis. En este estudio (18), Cayón y cols. no encuentran ningún cambio en la expresión genética de la IL-1 $\beta$, sin embargo, otros autores han encontrado en los pacientes con EHNA muy frecuentemente polimorfismos en este gen (34). Finalmente, los ratones deficientes en el factor PECAM-1 (platelet endothelial cell adhesion molecule-1), un factor supresor de las citoquinas proinflammatorias (TNF $\alpha$; MCP-1), desarrollan fácilmente EHNA (35).

Aunque en el estudio de Cayón y cols. no se hace referencia a genes relacionados con el estrés oxidativo, se podría esperar que los genes relacionados con este estrés o con la defensa frente a él pudieran estar modificados en la EHNA, ya que se considera que el estrés oxidativo juega un papel decisivo en la progresión de la esteatosis simple a esteatohepatitis (27). Ello podría explicar que sólo una minoría de sujetos con hígado graso evolucione a EHNA. La oxidación de los ácidos grasos es uno de los procesos donde se generan radicales reactivos derivados del oxígeno (ROS). Por ello, se ha estudiado el papel que pudieran jugar los genes que codifican enzimas implicadas en la oxidación de estos ácidos en la patogenia de la EHNA (3638). Los resultados que se han obtenido de esos estudios son difíciles de interpretar, ya que la interrupción de la $\beta$-oxidación, como ocurre en ausencia de acil-CoA oxidase, se sigue de una forma grave de EHNA con esteatosis microvesicular (39), pero las mismas consecuencias (40) tienen las mutaciones en el gen de la PPAR $\alpha$ (peroxisome proliferator-activated receptor), un factor de transcripción que aumenta la expresión genética de numerosas enzimas oxidativas de ácidos grasos (41). A pesar de ello, hay datos que indican que la pérdida de actividad de la PPAR $\alpha$ también se asocia con EHNA. Esto es lo que se encuentra en ratones carentes de este factor de transcripción (42). Por el contrario, el aumento de su actividad, estimulada por la adiponectina, protege frente a la esteatosis y EHNA $(43,44)$. Sin embargo, el papel 
de este factor queda oscurecido por estudios que muestran que el polimorfismo L162V, relacionado con un aumento de actividad de los PPAR $\alpha$, no influye sobre el desarrollo de EHNA (45). La magnitud del estrés oxidativo está determinada también por la presencia de hierro en las células y la eficacia de los sistemas antioxidantes. En relación con lo primero, algunos autores han referido que la mutación C282Y es mucho más frecuente en los pacientes con EHNA que en la población general (46). Sin embargo, este hallazgo no ha sido refrendado por otros investigadores (47), la concentración hepática de hierro no se relacionaba con la presencia de esas mutaciones y el grado de fibrosis hepática era independiente de la concentración de hierro en el tejido o de la existencia de mutaciones en el gen HFE. La homocisteína es también un factor inductor de estrés oxidativo y capaz de iniciar la peroxidación de los lípidos. Es bien sabido que las mutaciones del gen de la MTHTR (methylenetetrahydrofolate reductase) se acompañan de un aumento de la formación de homocisteína. Por esta razón se ha investigado si las mutaciones o polimorfismos del gen de esa enzima son más frecuentes en los pacientes con EHNA. Los resultados publicados hasta el momento son discrepantes, ya que mientras unos autores no encuentran aumentos en la frecuencia del polimorfismo MTHFR C677T en los pacientes con EHNA (48), otros encuentran que ese polimorfismo y el A1298C están muy aumentados en esos enfermos (49). En relación con los sistemas defensivos frente al estrés oxidativo, algunos autores han comunicado mutaciones en el gen de la superóxido dismutasa $(50,51)$ o que se asocian con los estados más avanzados de la EHNA. En el estudio de expresión genética realizado por Younossi y cols., se halló que algunos genes antioxidantes (catalasa, glutation S-transferasa A4) se encontraban sobreexpresados en los enfermos con EHNA (52). Sin duda se trata de una sobreexpresión reactiva a la exposición de las células al estrés oxidativo. Por el contrario, Sreekumar y cols. hallaron que el gen de la manganeso superóxido dismutasa está reprimido en enfermos con EHNA (53).

Se sabe muy poco sobre los factores genéticos responsables de que sólo algunos pacientes con EGHNA desarrollen fibrosis. Sin duda, el mantenimiento de la actividad inflamatoria hepática contribuye a ello. Como hemos visto más arriba, los factores genéticos pueden modificar la intensidad de esta respuesta favoreciendo o reprimiendo la producción de factores proinflamatorios. Pero, además, hay genes que determinan la respuesta fibrogénica individual y estos podrían ser los responsables de esos variables grados de fibrosis que se encuentran en los pacientes con EGHNA. En otras hepatopatías se ha encontrado que la presencia de fibrosis hepática está ligada a la existencia de polimorfismos en los genes de diversos factores implicados en la fibrogénesis. Este es el caso de los genes del TGFß1 (transforming growth factor) (54,55), CTGF (connective tissue growth factor), MMP-3 (matrix metalloproteinase), DDX5 (DEAD box polypeptide 5), CPT1A (carnitine palmitoyl transferase 1A) (56) y angiotensina II. Sólo contamos con un estudio en el que se ha comprobado que los sujetos obesos con expresión elevada de TGF $\beta 1$ y angiotensinógeno tienen una mayor tendencia a desarrollar fibrosis hepática (57). En el estudio de expresión genética realizado por Younossi y cols., se menciona que existe una sobreexpresión de los genes de la gelatinasa, una enzima que participa en la degradación de la matriz extracelular, y del versican, un componente de la matriz extracelular y, por ello, de la fibrosis (52). El estudio de Cayón y cols. aporta nuevos datos a este campo poco explorado. Su estudio confirma que los pacientes con EHNA y fibrosis tienen una sobreexpresión de los genes del TNF $\alpha$ y de la prohibitina, al igual que ocurre en los restantes pacientes con EHNA; sin embargo, este análisis añade que también lo están los genes del TGF $\beta$ (transforming growth factor), FGF (fibro- 
blast growth factor), CTGF (connective tissue growth factor) y del VEGF (vascular endotelial growth factor)] (18). La sobreexpresión de estos factores de crecimiento podía ser esperada en los pacientes con EHNA y fibrosis hepática, ya que son potentes activadores de la actividad fibrogénica y su expresión aumentada se ha encontrado en otras enfermedades que cursan con fibrosis hepática.

Un factor importante en la patogenia de la EGHNA es la retención de triglicéridos en el hígado. La abundante llegada de ácidos grasos al hígado procedente de la lipólisis de la grasa abdominal es uno de los factores que sin duda contribuye a la esteatosis hepática en los pacientes con síndrome metabólico. Por ello, se podría esperar que los factores genéticos que favorezcan la obesidad abdominal (58) también pudieran intervenir en el desarrollo de la EGHNA. En las primeras fases de la patogenia de esta enfermedad, la resistencia a la insulina juega un papel decisivo (27) y es bien conocido que el tipo 2 de diabetes mellitus está determinado genéticamente. En relación con ello, el estudio de Cayón y cols. demuestra que la expresión del gen de la IGFBP-1 (insulin-like growth factor binding protein-1) está aumentada en el hígado de los pacientes con EHNA (18). Se trata de un cambio que se ha encontrado ligado a la resistencia a la insulina $(59,60)$ y que coincide con lo comunicado por Sreekumar y cols. (53). Un aumento de esta proteína fijadora de IGF-1 determina que las tasas intracelulares de este IGF-1 libre se encuentren disminuidas y en consecuencia que aumente la resistencia a la insulina. A lo mismo pueden también contribuir los aumentos de expresión genética de diversas proteína reactantes de fase aguda ( $\alpha_{1}$ antitripsina, componente $\mathrm{C} 3 \mathrm{del}$ complemento, hepatocyte-derived fibrinogen-related protein 1) y de TNF $\alpha$ y su receptor (18). En otro lugar ya nos hemos referido al papel de esta última citoquina en la patogenia de la resistencia a la insulina (27). Es posible que si el estudio de Cayón y cols. hubiera incluido un grupo control formado por hígados de personas no obesas, sanas, habrían encontrado mayores diferencias en la expresión de genes implicados en patogenia de las primeras fases de la EGHNA. En este sentido, Younossi y cols. (52) hallaron sobreexpresión de varios genes relacionados con el metabolismo intrahepático de los lípidos [HMGCS2 (3-hydroxy-3-methylglutaryl-Coenzyme A synthase 2), ACSL4 (AcylCoA synthetase long-chain family member 4)].

Los ácidos grasos presentes en los hepatocitos son transformados en otros componentes celulares, son oxidados para producir energía o son excretados al plasma en forma de VLDL (very low density lipoprotein). Por ello, el grado de esteatosis hepática está influenciado también por el consumo de ácidos grasos y por la excreción de lipoproteínas. Una menor formación de VLDL, eventualmente de origen genético, podría favorecer la esteatosis hepática. Los resultados de algunos estudios publicados confirman que esta hipótesis es cierta. En efecto, en los pacientes con EGHNA se ha observado que es muy frecuente un polimorfismo en un único nucleótido (493G/T) del promotor del gen de la MTP (microsomal triglyceride transfer protein) que se acompaña de una menor actividad de esta proteína $(50,61)$. La MTP juega un papel muy importante en la formación y secreción de VLDL. Otro elemento muy importante en la formación de VLDL es la fosfatidilcolina (lecitina) (62), la cual se sintetiza a partir de la fosfatidiletanolamina con la contribución de la PEMT (phosphatidylethanolamine- $N$-methyltransferase). Los ratones con inactividad del gen de esta enzima y en los alimentados con colina desarrollan esteatosis hepática y EHNA $(63,64)$. Por ello, se ha buscado en pacientes con EGHNA la existencia de polimorfismos que pudieran estar ligados a una pérdida de actividad de esta enzima. En estos pacientes se ha hallado que el polimorfismo V175M, asociado a una baja actividad del gen de la PEMT, se encuentra significativamente aumentado en los pacientes con EGHNA (65). 
La nueva tecnología derivada del conocimiento del genoma humano, entre la que figura la utilizada por Cayon y cols. en este número de la Revista Española de Enfermedades Digestivas, está facilitando el avance en el conocimiento de los factores genéticos que pudieran intervenir en el desarrollo de las diferentes enfermedades, entre ellas la EGHNA, y que podrían justificar las susceptibilidades individuales a enfermar e informar sobre los mecanismos fisiopatológicos que pudieran estar implicados en su patogenia. El hallazgo de genes sobreexpresados, deprimidos o mutados o de polimorfismos ligados a hiper- o a inactividad permite suponer que las vías metabólicas donde sus productos proteicos ejercen sus funciones se encontrarán igualmente activadas o frenadas. Se trata de informaciones cuyo interés supera la mera curiosidad científica, ya que de ellas se pueden derivar decisiones con finalidad profiláctica o terapéutica. La aplicación de estas técnicas al estudio de la EGHNA es aún muy limitada $(53,66-68)$, entre otras razones por su costo aún excesivo, sin embargo, en los próximos años veremos que el número de aportaciones en este terreno se multiplicará, lo que contribuirá a aportar luz a esta enfermedad que sin duda será una de las plagas del siglo XXI.

\section{J. A. Solís-Herruzo y P. Solís-Muñoz \\ Centro de Investigación. Universidad Complutense. Hospital Universitario 12 de Octubre. Madrid}

\section{Bibliografía}

1. Musso G, Gambino R, De Michieli F, Cassader M, Rizzetto M, Faga E, et al. Dietary habits and their relations to insulin resistance and postprandial lipemia in non-alcoholic steatohepatitis. Hepatology 2003; 37: 909-16.

2. Zelber-Sagi S, Nitzan-Kaluski D, Goldsmith R, Webb M, Blendis L, Halpern Z, et al. Long term nutritional intake and the risk of non-alcoholic fatty liver disease (NAFLD): A population based study. J Hepatol 2007; 47: 711-7.

3. Zivkovic AM, German JB, Sanyal AJ. Comparative review of diets for the metabolic syndrome: Implications for nonalcoholic fatty liver disease. Am J Clin Nutr 2007; 86: 285-300.

4. DeFronzo RA, Ferrannini E. Insulin resistance. A multifaceted syndrome responsible for NIDDM, obesity, hypertension, dyslipidemia, and atherosclerotic cardiovascular disease. Diabetes Care 1991; 14: 173-94.

5. Ford ES, Giles WH, Mokdad AH. Increasing prevalence of the metabolic syndrome among U.S. adults. Diabetes Care 2004; 27: 2444-9.

6. Marchesini G, Bugianesi E, Forlani G, Cerrelli F, Lenzi M, Manini R, et al. Nonalcoholic fatty liver, steatohepatitis, and the metabolic syndrome. Hepatology 2003; 37: 917-23.

7. García-Monzón C, Martín-Pérez E, Iacono OL, Fernández-Bermejo M, Majano PL, Apolinario A, et al. Characterization of pathogenic and prognostic factors of nonalcoholic steatohepatitis associated with obesity. J Hepatol 2000; 33: 716-24.

8. Kallwitz ER, Herdegen J, Madura J, Jakate S, Cotler SJ. Liver enzymes and histology in obese patients with obstructive sleep apnea. J Clin Gastroenterol 2007; 41: 918-21.

9. Solga SF, Clark JM, Alkhuraishi AR, Torbenson M, Tabesh A, Schweitzer M, et al. Race and comorbid factors predict nonalcoholic fatty liver disease histopathology in severely obese patients. Surg Obes Relat Dis 2005; 1: 6-11.

10. Machado M, Marqués-Vidal P, Cortez-Pinto H. Hepatic histology in obese patients undergoing bariatric surgery. J Hepatol 2006; 45: 600-6.

11. Lima ML, Mourão SC, Diniz MT, Leite VH. Hepatic histopathology of patients with morbid obesity submitted to gastric bypass. Obes Surg 2005; 15: 661-9.

12. De Alwis N, Day CP. Non-alcoholic fatty liver disease: The mist gradually clears. J Hepatol 2008 (En prensa).

13. Struben V, Hespenheide EE, Caldwell SH. Nonalcoholic steatohepatitis and cryptogenic cirrosis within kindreds. Am J Med 2000; 108: 9-13.

14. Willner IR, Waters B, Patil SR, Reuben A, Morelli J, Riely CA. Ninety patients with non-alcoholic steatohepatitis; insulin resistance, familial tendency, and severity of disease. Am J Gastroenterol 2001; 96: 2957 61 .

15. Abdelmalek MF, Liu C, Shuster J, Nelson DR, Asal NR. Familial aggregation of insulin resistance in first-degree 
relatives of patients with nonalcoholic fatty liver disease. Clin Gastroenterol Hepatol 2006; 4: 1162-9.

16. Browning JD, Szczepaniak LS, Dobbins R, Nuremberg P, Horton JD, Cohen JC, et al. Prevalence of hepatic steatosis in an urban population in the United States: Impact of ethnicity. Hepatology 2004; 40: 1387-95.

17. Schwimmer JB, Deutsch R, Kahen T, Lavine JE, Stanley C, Behling C. Prevalence of fatty liver in children and adolescents. Pediatrics 2006; 118: 1388-93.

18. Cayón A, Crespo J, Guerra AR, Pons-Romero F. Expresión génica en pacientes con enfermedad hepática por depósito de grasa. Rev Esp Enferm Digest 2008; 100 (3): 212-8.

19. García-Ruiz I, Rodríguez-Juan C, Díaz-Sanjuán T, del Hoyo P, Colina F, Muñoz-Yagüe T, Solís-Herruzo JA. Uric acid and anti-TNF antibody improve mitochondrial dysfunction in ob/ob mice. Hepatology 2006; 44: 581-91.

20. Pérez-Carreras M, Del Hoyo P, Martín MA, Rubio JC, Martín A, Castellano G, et al. Defective hepatic mitochondrial respiratory chain in patients with nonalcoholic steatohepatitis. Hepatology 2003; 38: 999-1007.

21. Hui JM, Hodge A, Farrell GC, Kench JG, Kriketos A, George J. Beyond insulin resistance in NASH: TNF $\alpha$ or adiponectin? Hepatology 2004; 40: 46-54.

22. Li Z, Yang S, Lin H, Huang J, Watkins PA, Moser AB, et al. Probiotics and antibodies to TNF inhibit inflammatory activity and improve nonalcoholic fatty liver disease. Hepatology 2003; 37: 343-50.

23. Hotamisligil GS, Arner P, Caro JF, Atkinson RL, Spiegelman BM. Increased adipose tissue expression of tumor necrosis factor-alpha in human obesity and insulin resistance. J Clin Invest 1995; 95: 2409-15.

24. Miyazaki Y, Pipek R, Mandarino LJ, DeFronzo RA. Tumor necrosis factor alpha and insulin resistance in obese type 2 diabetic patients. Int J Obes Relat Metab Disord 2003; 27: 88-94.

25. Valenti L, Fracanzani AL, Dongiovanni P. Tumour necrosis factor a promotor polymorphisms and insulin resistance in non-alcoholic fatty liver disease. Gastroenterology 2002; 122: 274-80.

26. Day CP. CD14 promoter polymorphism associated with risk of NASH. J Hepatol 2002; 36 (Supl.): 21.

27. Solís Herruzo JA, García Ruiz I, Pérez Carreras M, Muñoz Yagüe MT. Non-alcoholic fatty liver disease. From insulin resistance to mitochondrial dysfunction. Rev Esp Enferm Dig 2006; 98: 844-74.

28. Feldstein AE, Canbay A, Angulo P, Taniai M, Burgart LJ, Lindor KD, et al. Hepatocyte apoptosis and Fas expression are prominent features of human non alcoholic steatohepatitis. Gastroenterology 2003; 125: 437-43.

29. Matsumaru K, Ji C, Kaplowitz N. Mechanisms for sensitization to TNF-induced apoptosis by acute glutathione depletion in murine hepatocytes. Hepatology 2003; 37: 1425-34.

30. Malhi H, Gores GJ, Lemasters JJ. Apoptosis and necrosis in the liver: A tale of two deaths? Hepatology 2006; 43: S31-S44.

31. García-Ruiz I, Rodríguez-Juan C, Díaz-Sanjuán T, Pérez-Carreras M, Solís-Muñoz P, Solís-Herruzo JA. El descenso de la actividad del complejo I de la cadena respiratoria mitocondrial en la esteatohepatitis noalcohólica está originado por un descenso de este complejo en las mitocondrias y no por un defecto en su ensamblaje. Gastroenterol Hepatol 2008; 31 (Supl. 1): 103A.

32. Santamaría E, Ávila MA, Latasa MU, Rubio A, Martín-Duce A, Lu SC, et al. Functional proteomics of nonalcoholic steatohepatitis: Mitochondrial proteins as targets of S-adenosylmethionine. PNAS 2003; 100: 306570 .

33. Aulak KS, Koeck T, Crabb JW, Stuehr DJ. This anion has probed to inhibit some mitochondrial protein. Am J Physiol Heart Circ Physiol 2004; 286: 30-8.

34. Nozaki Y, Saibara T, Nemoto Y, Ono M, Akisawa N, Iwasaki S, et al. Polymorphisms of interleukin-1 beta and beta 3-adrenergic receptor in Japanese patients with nonalcoholic steatohepatitis. Alcohol Clin Exp Res 2004; 28 (8 Supl. Proceedings): 106S-10S

35. Goel R, Boylan B, Gruman L, Newman PJ, North PE, Newman DK. The proinflammatory phenotype of PECAM-1-deficient mice results in atherogenic diet-induced steatohepatitis. Am J Physiol Gastrointest Liver Physiol 2007; 293: G1205-14.

36. Day CP. Pathogenesis of steatohepatitis. Best Pract Res Clin Gastroenterol 2002; 16: 663-78.

37. Sanyal AJ, Campbell-Sargent C, Mirshahi F, Rizzo WB, Contos MJ, Sterling RK, et al. Nonalcoholic steatohepatitis: Association of insulin resistance and mitochondrial abnormalities. Gastroenterology 2001; 120: 1183-92.

38. Miele L, Grieco A, Armuzzi A, Candelli M, Forgione A, Gasbarrini A, et al. Nonalcoholic steatohepatitis (NASH) and hepatic mitochondrial beta oxidation: Hepatic mitochondrial betaoxidation in patients with nonalcoholic steatohepatitis assessed by 13C-octanoate breath test. Am J Gastroenterol 2003; 98: 2335-65.

39. Fan CY, Pan J, Usuda N, Yeldandi AV, Rao MS, Reddy JK. Steatohepatitis, spontaneous peroxisome proliferation and liver tumors in mice lacking peroxisomal fatty acyl-CoA oxidase: Implications for peroxisome proliferator-activated receptor alpha natural ligand metabolism. J Biol Chem 1998; 273: 15639-45.

40. Merriman R, Aouizerat B, Molloy M. A genetic mutation in the peroxisome proliferator-activated receptor alpha gene in patients with non-alcoholic steatohepatitis. Hepatology 2001; 34: 441A.

41. Berger J, Moller D. The mechanism of action of PPARs. Annu Rev Med 2002; 53: 409-35.

42. Ip E, Farrell G, Robertson G, Hall P, Kirsch R, Leclercq I. Central role of PPARdependent hepatic lipid turnover in dietary steatohepatitis in mice. Hepatology 2003; 38: 123-32.

43. Xu A, Wang Y, Keshaw H, Xu LY, Lam KS, Cooper GJ. The fat-derived hormone adiponectin alleviates alcoholic and non-alcoholic fatty liver disease in mice. J Clin Invest 2003; 112: 91-100.

44. Yamauchi T, Kamon J, Minokoshi Y, Ito Y, Waki H, Uchida S, et al. Adiponectin stimulates glucose utilization and fatty-acid oxidation by activating AMP-activated protein kinase. Nat Med 2002; 8: 1288-95.

45. Verdi H, Koytak ES, Onder O, Ergül AA, Cinar K, Idilman R, et al. Peroxisome proliferator-activated receptor alpha L162V polymorphism in nonalcoholic steatohepatitis and genotype 1 hepatitis $\mathrm{C}$ virus-related liver steatosis. J Investig Med 2005; 53: 353-9.

46. George DK, Goldwurm S, MacDonald GA, Cowley LL, Walker NI, Ward PJ, et al. Increased hepatic iron concentration in non-alcoholic steatohepatitis is associated with increased fibrosis. Gastroenterology 1998; 114: 311-8.

47. Bugianesi E, Manzini P, D'Antico S, Vanni E, Longo F, Leone N, et al. Relative contribution of iron burden, 
HFE mutations and insulin resistance to fibrosis in nonalcoholic fatty liver. Hepatology 2004; 39: $179-87$.

48. Serin E, Güçlü M, Ataç FB, Verdi H, Kayaselçuk F, Ozer B, et al. Methylenetetrahydrofolate reductase C677T mutation and nonalcoholic fatty liver disease. Dig Dis Sci 2007; 52 (5): 1183-6.

49. Sazci A, Ergul E, Aygun C, Akpinar G, Senturk O, Hulagu S. Methylenetetrahydrofolate reductase gene polymorphisms in patients with nonalcoholic steatohepatitis (NASH). Cell Biochem Funct 2008 (en prensa).

50. Namikawa C, Shu-Ping Z, Vyselaar J, Nozaki Y, Nemoto Y, Ono M, et al. Polymorphisms of microsomal triglyceride transfer protein gene and manganese superoxide dismutase gene in nonalcoholic steatohepatitis. $\mathrm{J}$ Hepatol 2004; 40: 781-6.

51. Saksena S, Daly A, Leathart J, Day C. Manganese dependent superoxide dismutase (SOD2) targeting sequence polymorphism is associated with advanced fibrosis in patients with non-alcoholic fatty liver disease. $\mathrm{J}$ Hepatol 2003; 38 (Supl. 2): 47.

52. Younossi ZM, Gorreta F, Ong JP, Schlauch K, Giacco LD, Elariny H, et al. Hepatic gene expression in patients with obesity-related non-alcoholic steatohepatitis. Liver International 2005: 25: 760-71.

53. Sreekumar R, Rosado B, Rasmussen D, Charlton M. Hepatic gene expression in histologically progressive nonalcoholic steatohepatitis. Hepatology 2003; 38: 244-51.

54. Powell EE, Edwards-Smith CJ, Hay JL, Clouston AD, Crawford DH, Shorthouse C, et al. Host genetic factors influence disease progression in chronic hepatitis C. Hepatology 2000; 31: 828-33.

55. Osterreicher CH, Datz C, Stickel F, Hellerbrand C, Penz M, Hofer H, et al. TGFbetal gene polymorphism (codon $25 \mathrm{Arg}$ (Pro) affects progression to cirrhosis in patients with hereditary haemochromatosis). Cytokine 2005; 31: 142-8

56. Huang H, Shiffman ML, Cheung RC, Layden TJ, Friedman S, Abar OT, et al. Identification of two gene variants associated with risk of advanced fibrosis in patients with chronic hepatitis C. Gastroenterology 2006; 130: $1679-87$.

57. Dixon JB, Bhathal PS, Jonsson JR, Dixon AF, Powell EE, O'Brien PE. Pro-fibrotic polymorphisms predictive of advanced liver fibrosis in the severely obese. J Hepatol 2003; 39: 967-71.

58. WHO Expert Consultation. Appropriate body-mass index for Asian populations and its implications for policy and intervention strategies. Lancet 2004; 363: 157-63.

59. Mohamed-Ali V, Pinkney JH, Panahloo A, Cwyfan-Hughes S, Holly JM, Yudkin JS. Insulin-like growth factor binding protein-1 in NIDDM: Relationship with the insulin resistance syndrome. Clin Endocrinol 1999; 50: $221-8$

60. Shmueli E, Miell JP, Stewart M, Alberti KG, Record CO. High insulinlike growth factor binding protein 1 levels in cirrhosis: Link with insulin resistance. Hepatology 1996; 24: 127-33.

61. Bernard S, Touzet S, Personne I, Lapras V, Bondon PJ, Berthezène F, et al. Association between microsomal triglyceride transfer protein gene polymorphism and the biological features of liver steatosis in patients with type II diabetes. Diabetología 2000; 43: 995-9.

62. Yao ZM, Vance DE. The active synthesis of phosphatidylcholine is required for very low density lipoprotein secretion from rat hepatocytes. J Biol Chem 1988; 263: 2998-3004.

63. Zhu X, Song J, Mar MH, Edwards LJ, Zeisel SH. Phosphatidylethanolamine N-methyltransferase (PEMT) knockout mice have hepatic steatosis and abnormal hepatic choline metabolite concentrations despite ingesting a recommended dietary intake of choline. Biochem J 2003; 370: 987-93.

64. Dong H, Wang J, Li C, Hirose AL, Nozaki Y, Takahashi M. The phosphatidylethanolamine N-methyltransferase gene V175M single nucleotide polymorphism confers the susceptibility to NASH in Japanese population. J Hepatol 2007; 46: 915-20.

65. Song J, da Costa KA, Fischer LM, Kohlmeier M, Kwock L, Wang S, et al. Polymorphism of the PEMT gene and susceptibility to nonalcoholic fatty liver disease (NAFLD). FASEB J 2005; 19: 1266-71.

66. Ribeiro PS, Cortez-Pinto H, Sola S, Castro RE, Ramalho RM, Baptista A, et al. Hepatocyte apoptosis, expression of death receptors, and activation of NF-kappaB in the liver of nonalcoholic and alcoholic steatohepatitis patients. Am J Gastroenterol 2004; 99: 1708-17.

67. Crespo J, Cayón A, Fernández-Gil P, Hernández-Guerra M, Mayorga M, Domínguez-Díez A, et al. Gene expression of tumor necrosis factor alpha and TNFreceptors, p55 and p75, in nonalcoholic steatohepatitis patients. Hepatology 2001; 34: 1158-63.

68. Buque-García X. Caracterización del patrón de expresión génica en la esteatosis hepática asociada a la obesidad en hepatocitos de rata Zucker fa/fa. Tesis Doctoral. Universidad País Vasco; 2007. 